\title{
Multifractal models of small-scale solar magnetic fields
}

Article in The Astrophysical Journal · June 1994

DOI: $10.1086 / 174329$

CITATIONS

29

4 authors, including:

\section{Ana Cadavid}

California State University, Northridge

98 PUBLICATIONS 878 CITATIONS

SEE PROFILE

Alexander Ruzmaikin

California Institute of Technology

400 PUBLICATIONS 5,080 CITATIONS

SEE PROFILE
READS

19

\section{John K. Lawrence}

California State University, Northridge

147 PUBLICATIONS 1,092 CITATIONS

SEE PROFILE 


\title{
MULTIFRACTAL MODELS OF SMALL-SCALE SOLAR MAGNETIC FIELDS
}

\author{
A. C. Cadavid, J. K. Lawrence, A. A. Ruzmaikin, and A. Kayleng-Knight \\ San Fernando Observatory, Department of Physics and Astronomy, California State University, Northridge, CA 91330 \\ Received 1993 September 28; accepted 1993 December 9
}

\begin{abstract}
We generate, both analytically and numerically, artificial, two-dimensional images composed of a known self-similar, and thus multifractal, measure with added Gaussian white noise. These are used to interpret observed, line-of-sight, solar magnetic field distributions as noisy multifractals. The range of self-similar scaling of observed distributions is extended beyond that of previous work. Our interpretation of the data is then used to confront theoretical models for the generation of small-scale solar magnetic fields. We investigate the multifractal structure of the field generated by two-dimensional, random cell dynamos and find that selfsimilarity is relatively enhanced for more intermittent distributions and strong correlations between cells. An optimum value of the intercellular diffusion coefficient maximizes the degree of intermittency. The simulated field from a linear, kinematic, fast dynamo with two-dimensional, chaotic, "ABC" flow displays scaling properties resembling those of observed solar fields. We suggest that the chaotic element of this model is the crucial ingredient for the long-range correlations that lead to multifractal scaling.
\end{abstract}

Subject headings: methods: analytical - methods: numerical - MHD — Sun: magnetic fields

\section{INTRODUCTION}

The generation and dynamics of the large-scale, global solar magnetic field have been studied for decades in the context of mean-field dynamo theories (Parker 1979; Krause \& Rädler 1980; Zel'dovich, Ruzmaikin, \& Sokoloff 1983). At least a qualitative understanding of such phenomena as the 22 year solar magnetic cycle has been achieved. However, the small-scale photospheric fields, at or below the level of supergranules, are extremely intermittent both in spatial and temporal variations and are much less well understood. They are presumably governed by the stretch-twist-fold mechanism of the "fast" dynamo process in the turbulent convective motion of the plasma with its large magnetic Reynolds number $R_{m} \approx 10^{8}$. Quantitative description of the properties of the small-scale, photospheric fields is needed so that fast dynamo models can be confronted with the observations.

In previous work (Lawrence, Ruzmaikin, \& Cadavid 1993) we have found that the line-of-sight magnetic fields, in digital, photometric images of the solar photosphere, exhibit selfsimilarity when observed at varying levels of coarseness. Thus, they can be expressed in the form of a signed multifractal measure. Here we apply a new Monte Carlo procedure to improve counting statistics at coarser levels and extend the observed scaling range. The primary data were obtained at the San Fernando Observatory (SFO) with a spatial resolution $\geq 1$ " over active region-sized areas, and these have been supplemented by high resolution Lockheed magnetograms obtained at the Swedish Solar Observatory, La Palma. Altogether, self-similarity can be seen over a scaling range of 1.5 decades from $\sim 16^{\prime \prime}$ down to the limit of ground-based resolution at $\sim 0$."5.

Brandenburg et al. (1992) have carried out a full magnetohydrodynamic (MHD) simulation of field distribution with Reynolds numbers $R_{m} \approx 300$. They find that the modulus of the magnetic field has a multifractal structure at small scales. This result indicates that the self-similarity observed in the solar photospheric fields is in some way built into the equations of MHD convection. However, it still remains to identify the crucial ingredients of the theory which lead to these characteristics. Such an identification is necessary for actual understanding of the processes involved, as opposed to the ability to simulate them. Furthermore, the ability of full MHD codes to handle realistic values of $R_{m}$ is beyond present computer technology. A logical step is to construct the simplest physical model that can produce magnetic field distributions with a multifractal measure.

We study two simple models both analytically and by numerical simulation: images composed of Gaussian white noise and images containing a known multifractal generated by a multiplicative cascade process. These models strengthen our interpretation of the observed solar fields as noisy multifractals. They further offer the ability to quantify the noise content in an observed image.

Also studied are two physical models of fast dynamos that differ in their particular ingredients. These are the twodimensional random cellular dynamo model of Ruzmaikin, Liewer, \& Feynman (1994) and a two-dimensional, timedependent, chaotic "ABC" dynamo examined by Galloway $\&$ Proctor (1992). Because the solar data are necessarily in the form of two-dimensional images, we concentrate exclusively on models cast in the same format. The multifractal scaling properties of modeled images on two-dimensional grids are calculated and compared to the solar data. We wish to isolate the qualitative characteristics of the models, as well as optimal parameter values, that best reproduce the multifractal scaling and the small-scale singularity of the observed fields. The key ingredients appear to be intermittence in the field distributions and correlations on all spatial scales. Our results thus point to correlated chaotic motions of the solar plasma as an important feature for multifractal generation.

In $\S 2$ we review the concepts of a signed measure and cancellation exponent, and describe the box counting method based on a Monte Carlo technique that is used to calculate the singularity spectrum of the measure. We present in $\S 3$ an improved examination of the scaling properties of solar magnetic fields, based on a new Monte Carlo type box-counting 
technique. In $\S 4$ we apply analytical and box-counting methods to two mathematical field distributions (Gaussian white noise and an artificially generated multiplicative process) and to a mixture of the two. Then the scaling properties of two physical models, the random cell dynamo and the chaotic $A B C$ flow, are examined. Results are discussed and conclusions presented in $\S 5$.

\section{SCALING AND SELF-SIMILARITY OF A MEASURE}

We will pursue various aspects of scaling and self-similarity as they relate to the measure associated with the distribution of magnetic field. The concept of a measure is encountered frequently when conveying information about a system. Traditional examples are the distribution of monetary value on a supporting set of coins, the distribution of people on a set of city addresses, or the concentration of a metal under the surface of the Earth.

Here we will deal with $L \times L$ two-dimensional grids where the set of grid points represent the image pixels. A pixel corresponds to the minimum size of an observed feature and not its resolution. A measure representing the line-of-sight magnetic flux through each pixel can then be associated to a given grid. Unlike most familiar examples in the literature, here the lineof-sight magnetic flux takes both positive and negative values and thus constitutes a signed measure (Halmos 1962, chap. 6). We will investigate and confront the measures derived from three sources: observation in the form of solar magnetograms, generation by a known mathematical procedure, such as a Gaussian random process, and derivation from fast dynamo models of field generation in a turbulent, conducting medium.

\subsection{Box Counting Method and Multifractality}

To examine image scaling by the box counting method, the $L \times L$ pixel image is covered with uniform boxes of side $S$, and size $\epsilon \equiv S / L \ll 1$. The "coarse-grained" measure $\mu_{i}$ in the $i$ th box is calculated by a three step process. First we add the individual measures of each pixel and since these measures are signed, the oppositely signed contributions are allowed to cancel within a box. Then the absolute value of the measure is taken, and finally the measure is normalized to unit total value over the image. Thus the measure of coarse-grained boxes is always positive, even if the measures of the individual pixels are signed. We then study scaling properties of the measure, such as self-similarity, as the box size $\epsilon$ is varied.

To test the hypothesis of self-similarity the measure is cast in power-law scaling form:

$$
\mu\left(\alpha_{i}, \epsilon\right)=\epsilon^{\alpha_{i}},
$$

where $\alpha_{i}$ is the scaling "Hölder exponent" in each box.

To characterize the distribution of the measure for each coarse-graining scale $\epsilon$, we count the number $d N(\alpha, \epsilon)=n(\alpha, \epsilon)$ $d \alpha$ of boxes with the exponent $\alpha$ in bins of width $d \alpha$. In the present work the box counting is implemented via a Monte Carlo method which will be described in detail in $\S 3$. As it will be explained this technique improves the counting statistics of the large size boxes.

According to the criteria of Evertsz \& Mandelbrot (1992), if the number density $n(\alpha, \epsilon)$ also scales according to a power law:

$$
n(\alpha, \epsilon)=\phi(\epsilon) \epsilon^{-f(\alpha)}
$$

where $f(\alpha)$ is independent of $\epsilon$ in the limit $\epsilon \rightarrow 0$, and the function $\phi$ depends on $\epsilon$ only logarithmically, then the measure is self-similar and thus multifractal.

Even though $f(\alpha)$ is itself a regular function, it describes the singularity properties of the fields at small scales. It is thus referred to as the "singularity spectrum." Except for the slowly varying function $\phi(\epsilon)$ the singularity spectrum $f(\alpha)$ plays the role of the fractal dimension of the subset of boxes with singularity strength $\alpha$. In equation (2.2) essentially all of the contribution to $n(\alpha, \epsilon)$ in the limit $\epsilon \rightarrow 0$ comes at the maximum of the singularity spectrum $f\left(\alpha_{0}\right)$. Thus $D_{0}=f\left(\alpha_{0}\right)$ is the Hausdorff dimension of the supporting set of the distribution.

The normalization of the measure requires

$$
\int n(\alpha, \epsilon) \mu(\alpha, \epsilon) d \alpha=\int \phi(\epsilon) \epsilon^{\alpha-f(\alpha)} d \alpha=1
$$

(Van de Water \& Schram 1988; Meneveau \& Sreenivasan 1989). For this integral to remain finite but nonzero in the limit $\epsilon \rightarrow 0$ it is necessary that $f(\alpha) \leq \alpha$ for all $\alpha$ and that the equality hold for at least one value of $\alpha$. Hence the curve $f(\alpha)$ must lie below and be tangent to the "bisector" line $f(\alpha)=\alpha$ at a point that we call $\alpha_{1}$. When $\epsilon \neq 0$ it is not possible to maintain the normalization with $f$ independent of $\epsilon$ without including the slowly varying function $\phi(\epsilon)$. By use of Laplace's method (Meneveau \& Sreenivasan 1989) it is found that in the limit of small $\epsilon, \phi(\epsilon)$ is a power series in $[\ln (1 / \epsilon)]^{-1}$ :

$$
\phi(\epsilon)=C_{0}\left[\ln \left(\frac{1}{\epsilon}\right)\right]^{-1 / 2}\left\{1+C_{1}\left[\ln \left(\frac{1}{\epsilon}\right)\right]^{-1}+\cdots\right\},
$$

where $C_{0}, C_{1}, \ldots$ are independent of $\epsilon$ and depend in general on $f(\alpha)$ and its derivatives at the tangent point $\alpha_{1}=f\left(\alpha_{1}\right)$. For real solar data or modeled data we simply adjust the values of $C_{0}$ and $C_{1}$ to best make $f(\alpha)$ independent of $\epsilon$ over as broad a scaling range as possible. Thus, our $f(\alpha)$ is accurate to order $[\ln (1 / \epsilon)]^{-2}$, typically $\sim 3 \%$. Also typically $C_{0} \sim 1, C_{1} \sim-1$.

A common alternative approach to investigating multifractal measures is the method of moments leading to the generalized dimensions $D_{q}$ (Halsey et al. 1986; Chhabra et al. 1989). For completeness we give a brief description of the method of moments and explain its relation to our work.

Starting with the same measure as in equation (2.1), the generalized dimensions $D_{q}$ arise from the scaling exponents $\tau(q)$ of the $q$ th moments of the measure:

$$
\sum_{i} \mu_{i}^{q}(\epsilon) \propto \epsilon^{\tau(q)},
$$

where $D_{q} \equiv \tau(q) /(q-1)$ is defined to match the embedding Euclidean dimension (here 2) for a measure with uniform density. Although $q$ can be any real number, $D_{0}$ is the Hausdorff dimension $f\left(\alpha_{0}\right)$ of the supporting set of the measure. $D_{1}$ corresponds to the "information dimension" $f\left(\alpha_{1}\right)$, giving the scaling of the Shannon entropy of the measure. For a simple fractal $D_{q}=D_{0}$ for all $q$. In some work, the inequality of $D_{q}$ for selected values of $q$ is alone taken to indicate the presence of a multifractal; the Evertsz \& Mandelbrot (1992) criterion using $f(\alpha)$ is more intuitively meaningful. When $f(\alpha)$ and $D_{q}$ are smooth functions of $\alpha$ and $q$, respectively, and $f^{\prime \prime}(\alpha)<0$, they are related by the Legendre transformation

$$
\alpha=\frac{\partial \tau(q)}{\partial q} ; f[\alpha(q)]=q \alpha-\tau(q) .
$$

In the literature the method of moments is widely used, specially in theoretical studies of multifractals. In this paper, in 
which we are dealing with both real and simulated data, we find that the histogram method yields better results. As explained in the introduction, the main objective is to interpret the observed solar fields as noisy multifractals. To do this we have to be able to isolate the multifractal component and the white noise contribution in an unbiased fashion which is natural in the histogram method. In contrast, the method of moments yields very smooth curves in which the only evidence of the white noise component is their failure to converge for negative values of $q$.

Although in the histogram method there is some arbitrariness in adjusting the parameters $C_{0}$ and $C_{1}$ we find that the values do not change much from image to image. By the other side as explained by Evertsz \& Mandelbrot (1992), the calculation of the generalized dimensions $D_{q}$ from the slope of a curve of $\ln \left(\sum_{i} \mu_{i}^{q}\right) /(q-1)$ versus $\ln (\epsilon)$, through least-squares fitting, can only be justified if the curves of $\ln \left(\sum_{i} \mu_{i}^{q}\right)$ versus $\ln (\epsilon)$ are straight lines for a given $q$. In many cases a range of scales in which the technique is valid has to be specified.

We must add that in some preliminary studies we have found that for $q>0$ the generalized dimensions for the signed measure tend to be higher than those for the unsigned one. In addition this technique can yield direct information on the moment $q$ and corresponding $\alpha$ at which the multifractal characteristis tend to disappear. Indeed, the analysis via the method of moments can be a rather extensive subject that will be dealt with in future work.

\subsection{Cancellation Exponent}

Most physical processes are described by positive measures, such as probability measures. We are interested in signed measures that can take both positive and negative values. This concept was recently applied to dynamos with high Reynolds number turbulence by Ott et al. (1992) and Du \& Ott (1992). Such measures may in addition characterize a new type of singular behavior in which the magnetic flux changes its sign at arbitrarily small spatial scales.

Let

$$
\chi(\epsilon) \equiv \sum_{i}\left|\mu_{i}(\epsilon)\right|
$$

To characterize the singularity of the signs the concept of a "cancellation exponent" $\kappa$ is introduced as

$$
\kappa=\lim _{\epsilon \rightarrow 0} \frac{\ln [\chi(\epsilon)]}{\ln (1 / \epsilon)},
$$

where $\kappa \geq 0$ depending on the type of measure in consideration.

For a probability measure, $\sum_{i} \mu_{i} \equiv 1$, and $\kappa$ vanishes identically. If below some scale the magnetic field is given by a smooth density with a single sign, the quantity $\chi$ becomes constant and as the denominator grows, $\kappa \rightarrow 0$. For a sign-singular flux the numerator not only remains finite but grows in the limit of small scales and equation (2.8) gives a finite value for $\kappa$.

In practice the smallest scale size $\epsilon=1 / L$ is determined by the size of the lattice. We interpret $\kappa$ as the scaling exponent of $\chi$ over some scaling range and find the slope of a log-log plot of $\chi(\epsilon)$ versus $1 / \epsilon$ over that range.

\section{SCALING OF OBSERVED SOLAR MAGNETIC FIELDS}

In a previous work (Lawrence et al. 1993) we employed the concept of signed measures to analyze solar magnetic images.
Most of the data used were two-dimensional, digital, photometric images of line-of-sight magnetic field obtained at SFO (Chapman \& Walton 1989; Lawrence, Chapman, \& Walton 1991). Also used were Lockheed images obtained at the Swedish Solar Observatory, La Palma (Title et al. 1989).

Since magnetic flux is an extensive quantity while the magnetic field is a density, we associated the measure $\mu_{i}$ with the value of the net flux through $i$ th pixel. In order to deal with a positive definite measure we calculated the normalized absolute value of the flux:

$$
\mu_{i} \equiv \frac{\left|\Phi_{i}\right|}{\sum_{j}\left|\Phi_{j}\right|} .
$$

With this definition we apply the techniques described in $\S 2$.

When an $L \times L$ pixel image is rigidly partitioned into boxes as large as, say $\epsilon=\frac{1}{8}$, only $\epsilon^{-2}=64$ boxes are available to populate the $n(\alpha, \epsilon) d \alpha$ histogram of equation (2.2). Then poor counting statistics make the extension of self-similarity to this scale a practical impossibility. However, $\sim L^{2}>10^{4}$ locations of such boxes are available within the image. Our Monte Carlo counting procedure randomly samples these locations $N \gg \epsilon^{-2}$ times and then renormalizes the histogram to $\epsilon^{-2}$. Another advantage to this approach is that it is not necessary to rely on square pixel arrays; one can consider an $L_{1} \times L_{2}$ image with effective side $L=\left(L_{1} L_{2}\right)^{1 / 2}$. In addition, when only a small number of boxes are available to count, the histogram is extremely sensitive to accidental placement of solar features with respect to the partitioning. This random sampling method averages over many partitionings and allows a more faithful representation of the field distribution. Figure 1 shows the singularity spectrum corresponding to an SFO image of NOAA active region 5643 made on 1989 August 17. $N=50,000$ random samplings were used at each scale. The usable image measured $280 \times 460$ pixels giving an effective side $L=360$ pixels. The physical scale of the pixels was 0 "46

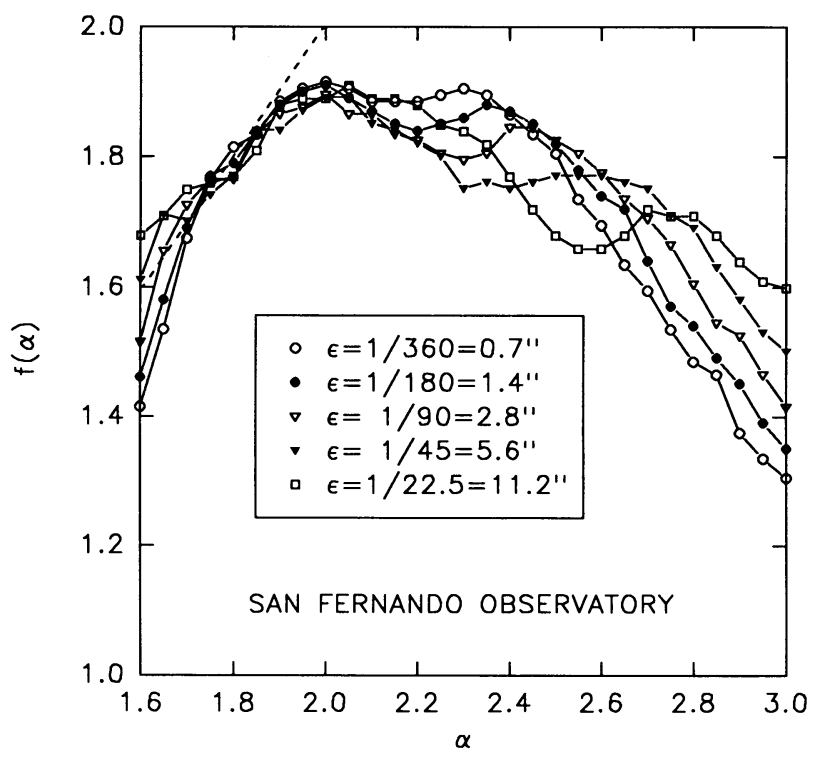

FIG. 1.- $f(\alpha)$ vs. $\alpha$ of a 1989 August 17, UT 22:59, SFO line-of-sight magnetic image including active region NOAA 5643 at heliocentric angle $\sim 5^{\circ}$. Pixel scales are 0 ".83 pixel $^{-1} \mathrm{NS}$ and 0.46 pixel $^{-1} \mathrm{EW} . C_{0}=1.1, C_{1}=-1.4$. The dashed line is the bisector $f(\alpha)=\alpha$. This is the same image analyzed in Lawrence et al. (1993) Fig. 4, with the counting statistics improved via the Monte Carlo method. 
EW and $0 " 83$ NS for an effective size 0.7 ; seeing is $\geq 1^{\prime \prime}$. With the choices $C_{0}=1.1, C_{1}=-1.4$ the $f(\alpha)$ curves converge for strong field values corresponding to $\alpha \leq 2.2$ over a scaling range from 0 ".7 to $11^{\prime \prime} 2$. We must note that this image was analyzed (Fig. 4) in the previous paper (Lawrence et al. 1993) using the "rigid" partition. The results here presented are an improvement over that original analysis. By examination of other SFO images and Lockheed/La Palma magnetograms with pixel size as small as 0.14 we can extend the scaling range to about 1.5 decades of scale from $\sim 0$ ". 5 to $\sim 16^{\prime \prime}$.

For the larger values of $\alpha$ in Figure 1, corresponding to the smaller magnetic flux values, the curves present a sequence of secondary peaks that appear farther to the right as $\epsilon$ increases. We will see below that this resembles the singularity spectrum of a multifractal with added white noise.

\section{SCALING OF MODEL MAGNETIC DISTRIBUTIONS}

We will consider the scaling properties of four models of magnetic images. The first two represent simple fundamental forms which can be treated both analytically and by simulation. They are not physical models, but are of use in interpreting the scaling properties of images. The last two models are attempts to represent the physics of small-scale field generation in a simple way. In these cases we attempt to use the interpreted observations to restrict the models.

\subsection{Gaussian White Noise}

Our renormalization scheme cancels elements of the measure with opposing signs in the coarse-graining procedure. Thus, an image whose pixels are entirely composed of white noise will display self-similarity. This is a consequence of the Central Limit Theorem, according to which the sum of a large number of random variables whose distributions have finite moments approaches a Gaussian random variable. This result is important because real data contain a random component, which may be instrumental noise, a Gaussian process in the solar field generation, or both. Further, understanding the correlations in solar magnetic field structures is advanced by the study of images whose pixels are completely uncorrelated. At this point we want to stress that in order to have $f(\alpha)$ curves that converge through a range of scales, we must use a signed measure since only then does the Central Limit Theorem apply. For the case of unsigned measures the $f(\alpha)$ curves do not converge as well as with the signed measure, even for values of $\alpha<2$, which as will be shown correspond to the multifractal contribution of the distribution.

The case of pure white noise not only will be studied via simulations but can also be treated analytically.

Let the Gaussian random two-dimensional image cells at coarse-graining scale $\epsilon$ contain a measure $b$ which is a random variable with the density:

$$
\rho(b, \epsilon)=\frac{1}{\pi \epsilon^{4}} \exp \left(-\frac{b^{2}}{\pi \epsilon^{4}}\right) .
$$

Here $\rho(b, \epsilon) d b$ is the number of cells of side $\epsilon$ with measure in between $b$ and $b+d b$. The normalization gives

$$
\int_{-\infty}^{\infty} d b \rho(b, \epsilon)=\frac{1}{\epsilon^{2}}
$$

where $1 / \epsilon^{2}$ is the total number of cells at each coarse graining level.
The total measure contained in $b$ is normalized according to

$$
\int_{-\infty}^{\infty} d b|b| \rho(b, \epsilon)=1 \text {. }
$$

To study the scaling we write

$$
\begin{gathered}
|b|=\epsilon^{\alpha} \\
n(\alpha, \epsilon)=\rho(b, \epsilon)\left|\frac{d b}{d \alpha}\right| .
\end{gathered}
$$

From this and equation (2.2) we construct an anlytical form for $f(\alpha, \epsilon) \rightarrow f(\alpha)$ :

$$
\begin{aligned}
f(\alpha, \epsilon) & \simeq(4-\alpha)-\frac{\epsilon^{2 \alpha-4}}{\pi \ln (1 / \epsilon)}+\frac{1}{\ln (1 / \epsilon)} \\
& \ln \left\{-\ln \left(\pi C_{0}\right)+\ln \left[1-\frac{C_{1}}{\ln (1 / \epsilon)}+\cdots\right]+\frac{3}{2} \ln \left[\ln \left(\frac{1}{\epsilon}\right)\right]\right\} .
\end{aligned}
$$

When $\epsilon$ is finite $f(\alpha)$ extends above the bisector and converges only logarithmically to a single curve as $\epsilon \rightarrow 0$. The limiting curve is

$$
f(\alpha)=4-\alpha(\alpha \geq 2) ; \lim _{\epsilon \rightarrow 0} f(\alpha) \rightarrow-\infty(\alpha<2) .
$$

Plots of these limiting curves are shown in Figure 2 as solid lines. The limiting curve is a heavy solid line.

For white noise the cancellation exponent $\kappa \rightarrow 1$ as $\epsilon \rightarrow 0$.

\subsection{Multiplicative Multifractal Measure}

A simple procedure, described by Meakin (1987) as an elementary model for turbulence, generates two-dimensional measures with self-similarity. It starts with a unit measure, uniformly distributed on a square. The square is partitioned into four equal squares, and the measure in each is multiplied

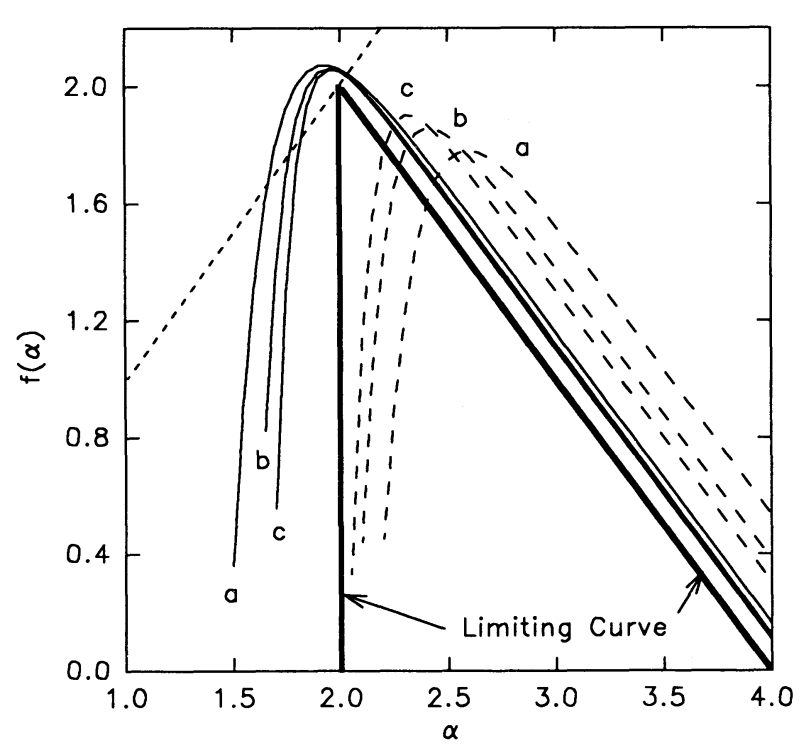

Fig. 2. $-f(\alpha)$ vs. $\alpha$ for an analytically generated Gaussian distribution. Scales are $(a) 1 / 360,(b) 1 / 90,(c) 1 / 22.5$. Solid lines are for $p=m=1.0$; dashed lines are for $p=0.4, m=0.05$, the heavy solid line is the limiting curve for $\epsilon \equiv 0$ for all $p, m$. In addition $C_{0}=1, C_{1}=-1$. 
by a different one of four factors $\xi_{i}$, where $0 \leq \xi_{i} \leq 1$ for $i=1$, $2,3,4$. Each of the four squares is subsequently partitioned, and the same four factors $\xi_{i}$ are randomly allocated (without replacement) among the parts. After $N$ repetitions, the original square is composed of $2^{N} \times 2^{N}$ pixels, each with some measure $\mu=\xi_{1}^{a} \xi_{2}^{b} \xi_{3}^{c} \xi_{4}^{d}$, where $a+b+c+d=N$.

In the limit $N \rightarrow \infty$ the generalized dimensions of this measure can be computed exactly (Martinez 1991):

$$
D_{q}=\frac{\tau(q)}{(q-1)}=\frac{\ln \left(\sum_{i} w_{i}^{q}\right)}{(q-1) \ln (1 / 2)},
$$

where $w_{i} \equiv \xi_{i} / \sum_{j} \xi_{j}$. Putting this in the Legendre transformation of equation (2.6) gives an analytical expression for the singularity spectrum in parametric form over $q$ :

$$
\alpha=\frac{\sum_{i} w_{i}^{q} \ln w_{i}}{\ln (1 / 2) \sum_{i} w_{i}^{q}} ; f[\alpha(q)]=q \alpha-\frac{\ln \sum_{i} w_{i}^{q}}{\ln (1 / 2)} .
$$

For example the choices $\xi_{1}=1, \xi_{2}=0.6, \xi_{3}=0.2, \xi_{4}=0$ give a Hausdorff or box dimension $D_{0}=\ln 3 / \ln 2 \approx 1.585$. (The only values of $D_{0}$ available to this model are $\left.1,1.585,2\right)$. The corresponding $f(\alpha)$ curve is shown as the heavy line in Figure 3.

This procedure cannot exactly model the generation of solar magnetic fields; it produces a positive definite rather than a signed measure. However it possesses a property which may underlie the self similar nature of the solar fields: a kind of correlation on all scales. In the present case, this correlation is provided by the application of the same rules $\xi_{i}$ at all levels of the partitioning.

Because this measure is strictly positive the cancellation exponent $\kappa$ is identically zero.

\subsection{Interpretation of Observed Fields as Noisy Multifractals}

For comparison to the solar data, we have generated a measure, on a $256 \times 256(N=8)$ array, composed of the sum of the above multiplicative multifractal containing $95 \%$ of the measure and white noise containing $5 \%$.

To encompass this case it is necessary to renormalize the amplitude and variance of the Gaussian density in equation

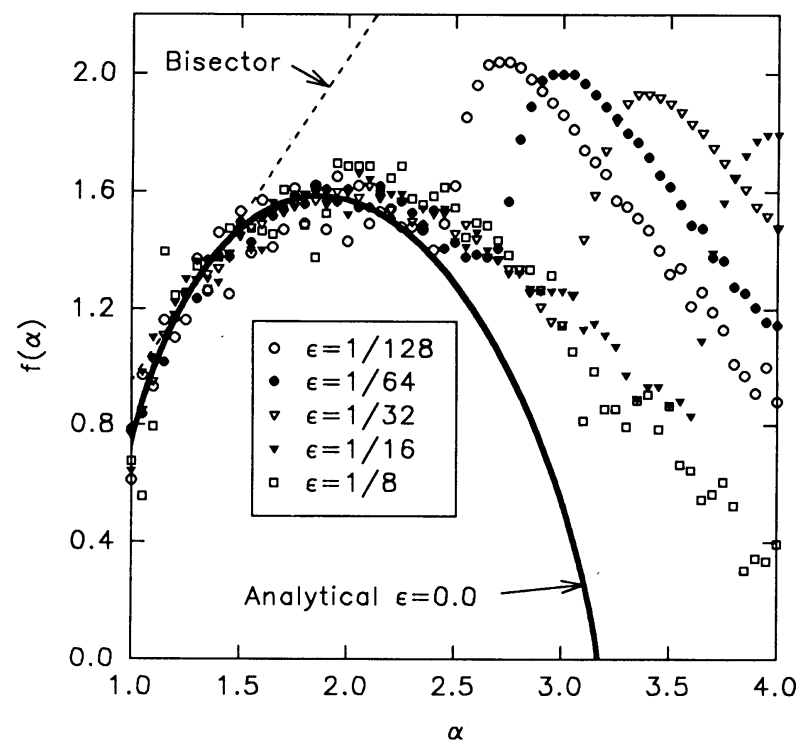

FIG. 3. $-f(\alpha)$ vs. $\alpha$ for a multiplicative multifractal with the measure multiplied by 0.95 and added white noise with $p=1$ and $m=0.5$. The solid line corresponds to the limiting curve for the pure multifractal for $\epsilon \rightarrow 0$. Also $C_{0}=0.7, C_{1}=-0.8$.
(4.1) to the form

$$
\rho(b, \epsilon)=\frac{p^{2}}{\pi m \epsilon^{4}} \exp \left(-\frac{p^{2} b^{2}}{\pi m^{2} \epsilon^{4}}\right),
$$

where $p \leq 1$ represents the fraction of image cells in which the noise is located, and $m \leq 1$ represents the fraction of the total measure contained in the noise. For $p<1$ and/or $m<1$, the $f(\alpha)$ curves are displaced relative to one another in a cascade for increasing values of $\epsilon$. This is illustrated analytically by the dashed curves in Figure 2. To the generated multifractal with $\xi_{i}$ chosen as above and with the measure multiplied by 0.95 , we added white noise generated by the rescaled density equation (4.9) with $p=1.0, m=0.05$.

The box-counting procedure gives the $f(\alpha)$ shown in Figure 3. For $N=8$ the values of $\mu$ taken by the generated multifractal are sufficiently discrete to lead to aliasing in our $\alpha$ bins for small $\epsilon$. With a fit $C_{0}=0.7 ; C_{1}=-0.8$ the multifractal produces a single $f(\alpha)$ curve over a range of scales. The small $\alpha$, strong-field, multifractal part of the $f(\alpha)$ curve converges, but the large $\alpha$, weak field curves rise to a cascade of peaks representing noise. This form matches qualitatively the form of $f(\alpha)$ found for solar magnetic data and shown in Figure 1. A quantitative comparison of the curves allows us to estimate the extent and the strength (e.g., $p$ and $m$ ) of the noise components in the data images.

We suggest that the strong field structures that correspond to the left-hand side of Figure 1 are multifractal (i.e., selfsimilar) in nature. The peaks on the right-hand side represent a Gaussian random variable which might contain quiet Sun intermittent fields, observational noise, or both. White noise singularity spectra with $p=0.4$ and $m=0.05$ fit the curves for all scales. These choices correspond to the dashed curves in Figure 2. The small value for $m$ implies that only a small fraction of the measure is random.

The multifractal part of the $f(\alpha)$ curves has a maximum $f_{\max } \approx 1.85$ at $\alpha \approx 2$. Further, $f(\alpha)$ is tangent to the bisector at $f\left(\alpha_{1}\right) \approx 1.7$.

The cancellation exponent for the image of Figure 1 was calculated to be $\kappa=0.01$. For a San Ferando Observatory quiet sun image, containing only network magnetic fields, the multifractal form was reproduced, the Gaussian component corresponded to $p=0.5, m=0.3$, and the cancellation exponent was found to be $\kappa=0.12$. For a Lockheed/La Palma active region magnetogram we found the same form with $p=1.0, m=0.18$, and $\kappa=0.03$. Finally, the scaling of the cancellation exponent $\kappa$ for the generated multifractal-plus-noise model is poor (the log-log plot of $\chi$ vs. $1 / \epsilon$ is not very linear) unless the Gaussian component is large. The scaling of $\kappa$ is somewhat better for the solar data, no doubt because the magnetic measure itself is signed, and not positive definite as in our example. This last point must be kept in mind in comparing the observed solar magnetic fields to the generated multifractal. Although the true solar fields (as opposed to the noise) show both signs, on the small scale one sign may dominate in individual cells, thus simulating the positive definite measure.

\subsection{Two-Dimensional Random Cell Dynamo}

As an elementary physical description of small-scale magnetic field fluctuations Poezd, Ruzmaikin, \& Sokoloff (1992) for one dimension and Ruzmaikin et al. (1994) for two dimensions introduced a cellular model for a dynamo in a random velocity field. It assumes that the basic processes of field amplification and reconnection (e.g., the "stretch-twist-fold" 
mechanism) take place in localized regions of space that can be considered as independent cells. To account for turbulent diffusion, an interaction between the cells is introduced through a random walk mechanism. To match the solar data, we focus on the two-dimensional cellular dynamo model described below.

A surface is partitioned into cells where the independent dynamo processes take place. Starting with a seed magnetic field the stretch-twist-fold mechanism then randomly produces enhancement or reconnection. The two-dimensional system evolves in each time step according to

$$
B^{(n+1)}(i, j)=q_{i, j} p_{i, j} f(B) B^{(n)}(i, j),
$$

where $i, j$ are Cartesian spatial cell indices and $n$ is the time step index.

The parameter $p_{i, j}$ is given by

$$
p_{i, j}=e^{\xi}
$$

where $\xi$ is a normally distributed random variable with $\langle\xi\rangle=0,\left\langle\xi^{2}\right\rangle=1$. The sign

$$
q_{i, j}= \pm 1
$$

also is generated randomly with equal probabilities.

The function $f(B)$ simulates a feedback field damping mechanism that can be of either a prompt or delayed type depending on whether the field value before or after the application of $p$ and $q$ is used:

$$
f(B)=\frac{1}{1+B^{2}} .
$$

The cells interact via a random walk in two dimensions. This is regulated by the coefficient $0 \leq \eta \leq \frac{1}{4}$, which expresses the probability of communication between a cell and its four nearest neighbors:

$$
\begin{aligned}
& B^{(n+1)}(i, j)=(1-4 \eta) B^{(n)}(i, j) \\
& +\eta\left[B^{(n)}(i+1, j)+B^{(n)}(i-1, j)+B^{(n)}(i, j+1)+B^{(n)}(i, j-1)\right] .
\end{aligned}
$$

In the limit $\Delta x, \Delta y \rightarrow 0$ as $\Delta t \rightarrow 0$, equation (4.14) reduces to the diffusion equation with coefficient $\mathscr{D}$ :

$$
\mathscr{D}=\lim _{\Delta x, \Delta t \rightarrow 0} \eta \frac{(\Delta x)^{2}}{\Delta t} .
$$

Obviously, the value of the diffusion coefficient will depend on how the limiting process is performed. For simplicity, we will refer to $\eta$ as the diffusion coefficient.

If desired, an additional correlation between cells can be controlled with a parameter $0<\zeta<1$ by replacing the number $p_{i, j}$ in equation (4.10) by, for example,

$$
r_{i, j}=(1-\zeta) p_{i, j}+\frac{1}{4} \zeta\left(p_{i+1, j}+p_{i-1, j}+p_{i, j+1}+p_{i, j-1}\right) \text {. }
$$

In implementing the simulation, all cells are initialized to $B^{(0)}(i, j)=1, i, j=1, \ldots, N$. The evolution in time is followed by first applying the diffusion type equation (4.14) followed by the random transformation (4.10).

A statistical steady state is reached when the variance $\left\langle B^{2}\right\rangle$ and the flatness

$$
F=\frac{\left\langle B^{4}\right\rangle}{\left\langle B^{2}\right\rangle^{2}}
$$

no longer change systematically in time. The averages in angle brackets are taken both over space and time. The flatness mea- sures the degree of intermittency of the distribution, as compared to a purely Gaussian distribution with $F=3$. The flatness is $F>3$ for an intermittent distribution with enhanced tails. For the observed solar fields in Figure 1 we find a flatness $F \approx 14$; several solar images give values in the range $6 \leq F \leq 50$. For different values of $\eta$ and $\zeta$ the random cell model requires different numbers of time steps $T$ to reach a statistically steady state. The values of $F$ and $\left\langle B^{2}\right\rangle$ only stabilize fully for the cases with $\eta \neq 0$ and $\zeta=0$ for $T>5000$ steps. However, $F$ and $\left\langle B^{2}\right\rangle$ as functions of $\eta$ always yield essentially the same shape curve, provided $T>1000$ steps. Likewise, the singularity spectra and the cancellation exponent do not vary significantly for $T>1000$ steps.

When both $\eta$ and $\zeta$ are nonzero, the magnitudes of neighboring generated fields are highly correlated, but their relative signs are random. This leads to strong cancellation, and the generated fields tend to zero with time. In what follows, we concentrate on the case $\eta>0, \zeta=0$. The other cases do not differ significantly and will be touched on only briefly. For a delayed feedback, the calculated values of the flatness $F$ are larger than the observed ones by several orders of magnitude, and we thus reject this case as contrary to the data. For the prompt feedback the flatness is larger than, but comparable to, that of the data.

Figure 4 shows $F,\left\langle B^{2}\right\rangle$ and $\kappa$ as functions of the diffusion coefficient $\eta$ with the values taken at $T=5000$ steps for prompt feedback. It is an intriguing result that there appears to be an optimum value of the diffusion coefficient, leading to

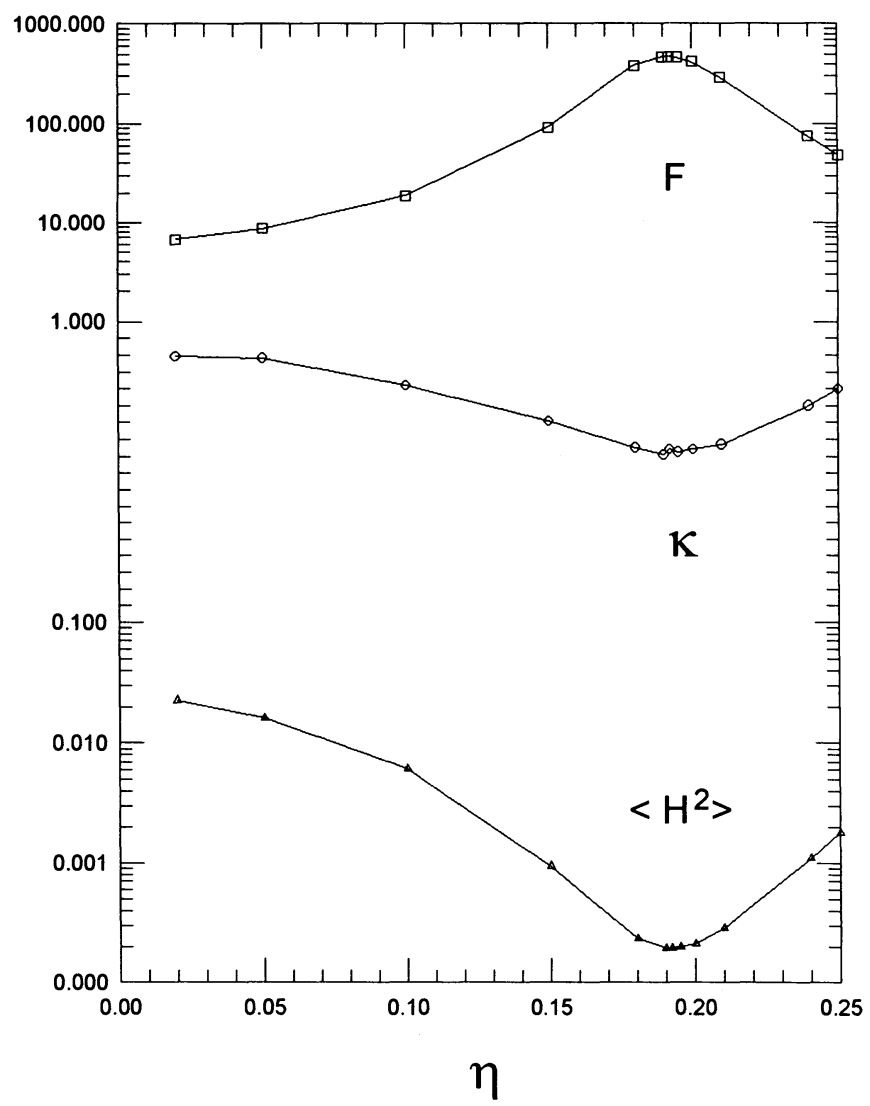

Fig. 4.-Variation of $F, \kappa$, and $\left\langle B^{2}\right\rangle$ with $\eta$ for the magnetic field generated via the cellular dynamo for the prompt nonlinearity and $\zeta=0$ at $T=5000$. The values are taken for $256 \times 256$ pixel $^{2}$ samples. 


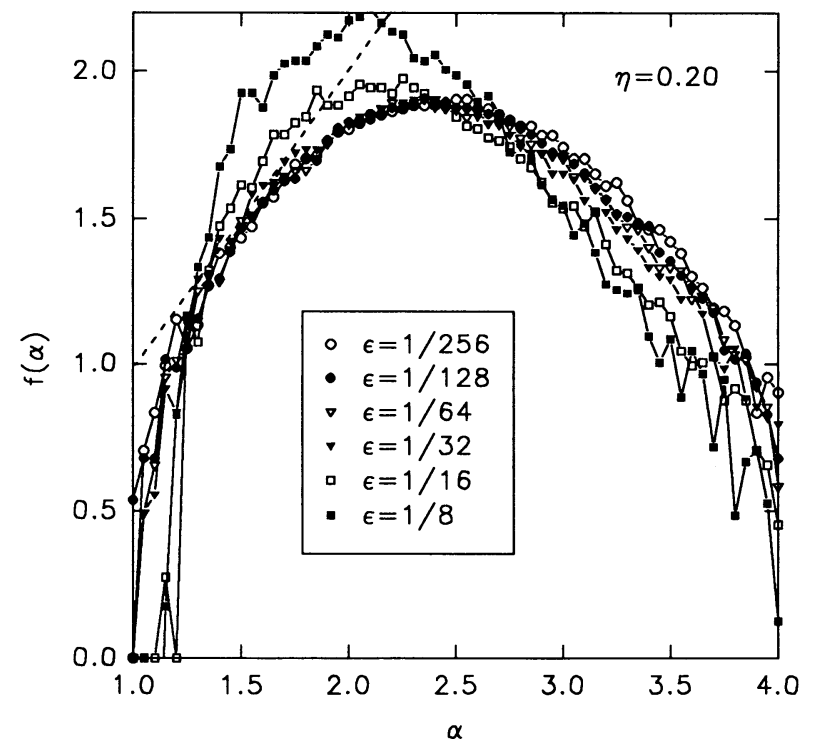

Fig. 5. $-f(\alpha)$ vs. $\alpha$ for a $256 \times 256$ pixel $^{2}$ sample for the magnetic field generated via the cellular dynamo for the prompt nonlinearity, $\eta=0.20$ and $\zeta=0.0$ at $T=2000$. $C_{0}=0.8, C_{1}=-1.4$. The dashed line is the bisector $f(\alpha)=\alpha$.

generated images with maximized intermittent, as opposed to random, behavior. The values of $F$ have a maximum and of $\kappa$ and $\left\langle B_{\min }^{2}\right\rangle$ have a minimum for $\eta \approx 0.2$. The smallest values for the cancellation exponent $0.5<\kappa<0.6$ are considerably larger than the value of $\kappa$ found for even a quiet Sun image.

Figure 5 displays the singularity spectrum near the optimum value at $\eta=0.2$ at $T=2000$ steps. The choice $C_{0}=0.8$ and $C_{1}=-1.4$ brings the curves for the smallest four scales into congruence. For the larger scales, $\epsilon \geq 1 / 16$, the curves approach Gaussian ones with $p \approx 1, m \approx 1$; their slopes for large $\alpha$ approach -1 , and their maxima extend above the bisector. We interpret this to mean that over distances greater than $\sim 8$ pixels the image features become uncorrelated.

The maxima for the smaller scales occur at $\alpha \approx 2.4$, while the multifractal model, the chaotic "ABC" dynamo model below, and the solar data have maxima for $\alpha$ close to 2.0. Although in all cases $f_{\max }$ is less than 2, this does not necessarily indicate that the dimension of the support is less than 2 , since in the multifractal analysis there is a low cutoff. Apart from this, in the observed solar data there is a low cutoff of approximately $15 \mathrm{G}$, while in the simulations although the magnetic field is continuous and in principle occupies the whole support, we have a finite sensitivity since some of the low end values are many orders of magnitude smaller than the peak magnetic fields. We must note that in earlier studies (Ruzmaikin, Sokoloff, \& Tarbell 1991) it was shown that for quiet Sun regions $D_{0}$ depends only slightly on the level of the cutoff and the actual dimension of the support may be less than 2 .

Since for both $\eta<0.19$ and $\eta>0.2$ the cancellation exponent is closer to 1 , we expect that these cases should have a larger Gaussian component. Figure 6 shows the singularity spectrum for $\eta=0.24$ at $T=1000$ steps, with $C_{0}=0.8$ and $C_{1}=-1.2$. The $f(\alpha)$ curves do not match for any scale; their maxima occur for different values of $\alpha$. They quickly approach the Gaussian random form with increasing $\epsilon$. A matching calculation with very small diffusion coefficient $\eta=0.02$ gives a qualitatively similar result.
Two underlying effects appear to be at play in these results. The Central Limit Theorem tells us that the sum of many independent random variables with finite moments approaches a Gaussian random variable. Thus the coarsegraining process causes the $f(\alpha)$ curves to look more and more Gaussian at large scales. By comparing the flatness we find that the distributions corresponding to $\eta=0.02$ and $\eta=0.24$ tend to be more nearly Gaussian than the one generated with $\eta=0.2$, which tends to be more intermittent, even though it does not have the strongest communication between cells. An intermittent distribution has strong, large field tails. Random walk communication among cells has two effects. First, the strong fields are spread out, which populates the distribution tails and increases intermittence. Second, however, the large field strengths are correspondingly reduced, which truncates the tails and reduces intermittence. The balance between these effects appears responsible for the optimally intermittent case for $\eta=0.2$. The corresponding $f(\alpha)$ plots suggest that, in the context of the cellular dynamo model, the more intermittent the distribution the more it "looks" like a multifractal.

When nonvanishing cellular correlation $\zeta$ was considered, we found that if we included a random sign together with the diffusion mechanism the magnetic field died at $\sim 200$ time steps. For the prompt nonlinearity with random sign and $\eta=0$ at $T=2000$ time steps we found: for $\zeta=0.2, F=2.1$ and $\kappa=0.99 ; \zeta=0.5, F=1.3$ and $\kappa=1$. With $F<3$ these cases are even less intermittent than the Gaussian. For the delayed nonlinearity with random sign and $\eta=0$ at $T=2000$ time steps we found: for $\zeta=0.2, F=81$ and $\kappa=0.91$; for $\zeta=0.5, F=18$ and $\kappa=0.97$. In the case with delayed nonlinearity and $\zeta=0.2$, the $f(\alpha)$ curves showed a sudden "phase transition" from self-similar behavior for $\epsilon=1 / 256,1 / 128$ to random Gaussian behavior for $\epsilon \geq 1 / 64$. Since the spatial correlation only relates the four nearest neighbor pixels, when the size of the coarse-graining box exceeds this correlation length we have once again a system of independent cells, and the measure behaves as a Gaussian. Note that for $\eta=0, \zeta>0$

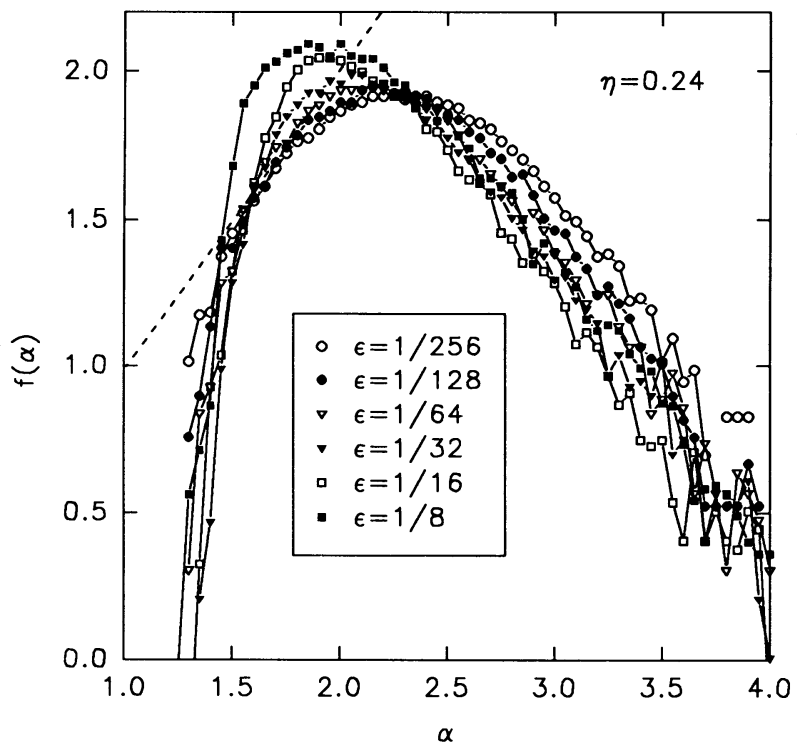

FIG. 6. $-f(\alpha)$ vs. $\alpha$ for a $256 \times 256$ pixel $^{2} p x^{2}$ sample for the magnetic field generated via the cellular dynamo for the prompt nonlinearity, $\eta=0.24$ and $\zeta=0.0$ at $T=1000$. $C_{0}=0.8, C_{1}=-1.2$. The dashed line is the bisector $f(\alpha)=\alpha$. 
the delayed feedback gives a more realistic flatness than does the prompt.

\subsection{Fast Kinematic Dynamo with Chaotic Flow}

This model differs strongly from the random cell dynamo in that it explicitly describes the stretch-twist-fold mechanism through a prescribed, but chaotic, plasma flow field. The kinematic dynamo problem consists in solving the induction equation for $B$ with a given velocity field $v$ and a magnetic Reynolds number $R_{m}$ :

$$
\frac{\partial B}{\partial t}=\nabla \times(v \times B)+\frac{1}{R_{m}} \nabla^{2} B .
$$

Fast dynamo action is obtained for flow fields which produce a growth rate of $B$ which remains finite as $R_{m} \rightarrow \infty$, and thus does not depend on field line reconnections. It has been suggested that chaotic flows in which the separation between neighboring points grows exponentially should be good candidates for fast dynamos. This should lead to solutions of the kinematic induction equation in which $\boldsymbol{B}$ grows exponentially.

A simplified Arnold, Beltrami, Childress (ABC) flow has been investigated by Galloway \& Proctor (1992). The dependence of the flow field on only two dimensions permitted computations on a relatively large grid with magnetic Reynolds number as large as $R_{m} \sim 2000$. The flow remains chaotic, however, because it is time-dependent. The velocity field given by

$$
\begin{aligned}
& v=[A \sin (z+\sin \omega t)+C \cos (y+\cos \omega t), \\
& A \cos (z+\sin \omega t), C \sin (y+\cos \omega t)]
\end{aligned}
$$

not only presented large chaotic regions of the phase space but also tended to produce an additional "circular polarization" on the original $\mathrm{ABC}$ flow. The solution technique developed in an earlier work (Galloway \& Frisch 1986) consists of expanding the magnetic field in a triple Fourier series, substituting into the induction equation, and letting the system evolve by using second-order time stepping. The results studied by us used $A=C=(3 / 2)^{1 / 2}, \omega=1$, and correspond to $B_{x}$ in the $y, z$ plane at $x=0$ for a magnetic Reynolds number of 2000 . These were presented in the form of a $481 \times 481$ pixel, digital "image" which was input as data into our scaling histogram procedure. This digital image was provided to us by David Galloway.

Before we perform the analysis, a $2 \times 2$ pixel reduction is applied to the images. In the reduced $240 \times 240$ pixel image each pixell is derived from a block of four, discarding the lowest and highest values and averaging the other two. Figure 7 gives $f(\alpha)$ for the data generated via this flow with parameters $C_{0}=1$ and $C_{1}=-1.5$. Over four scales of 2 the curves display the familiar combination of two distinct structures: one corresponding to the strong, self-similar fields that contribute to the left hand side of the curve, the other given by a random variable that presents a series of secondary maxima. We interpret this as representing a multifractal distribution with added noise. The secondary maxima occur for large values of $\alpha$, and the corresponding values of $p$ and $m$ indicate that only a small fraction of the pixels carry the random variable and that only an extremely small fraction of the measure is contained in it. The noise may be an artifact of the Fourier analysis of the flow. For practical purposes, the measure is contained in the self similar active region fields described by the left-hand portion of

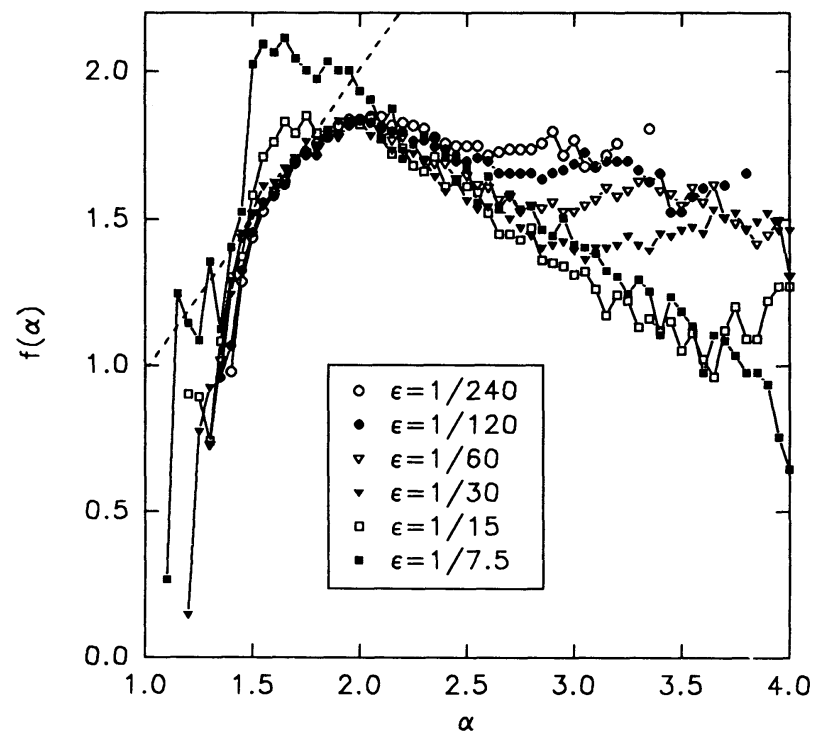

Fig. 7. $-f(\alpha)$ vs. $\alpha$ for a $256 \times 256$ pixel $^{2}$ sample for the magnetic field generated with the fast dynamo with "ABC" flow (Galloway \& Proctor 1992). $C_{0}=1, C_{1}=-1.5$. The dashed line is the bisector $f(\alpha)=\alpha$.

the singularity spectrum. The maximum, $f_{\max } \approx 1.8$ occurs for $\alpha \sim 2$.

For scales $\geq 1 / 16$ the curves begin to resemble those for white noise: they extend across the bisector and the slopes $\rightarrow-1$ for large $\alpha$.

The cancellation exponent is found to be $\kappa=0.11$, comparable to that for observed solar fields. The calculated flatness is $F=29$, also comparable to observed values.

\section{DISCUSSION AND CONCLUSIONS}

The Monte Carlo method we have used in our multifractal box-counting analysis allows the accumulation of good counting statistics even for relatively large box sizes. Further, this random sampling procedure averages over many box partitionings of images and allows more accurate representation of the field distribution. By applying this to a variety of SFO and Lockheed/La Palma magnetic images, we can observe selfsimilarity from the limit of terrestrial seeing at $\sim 0.5$ up to $\sim 16^{\prime \prime}$. This represents about 1.5 decades of scaling, a significant increase over our previous work (Lawrence et al. 1993).

Our treatment of signed measures is such that Gaussian white noise is self-similar. Analytical study of this property is useful in interpreting both solar data and modeled images. For example, images generated by the random cell dynamo resemble noise at large scales. Similarly, peak cascades in $f(\alpha)$ curves for large $\alpha$ are found to signify noise in the image. We have hopes that our ability to quantify the noise content may have applications to image restoration.

The analytical treatment of the singularity spectrum presented here, assists in the interpretation of solar field images as noisy multifractals. It also offers a clue to the origin of the multifractality of the fields in that it applies a single partitioning rule at all scales in the image.

The random cell dynamo model without correlation between the cells bears a closer resemblance to random variable images than to the solar field images. This resemblance is seen in the derived $f(\alpha)$ spectra and in the large values of the cancellation coefficients. The Central Limit Theorem tells us 
that the sum of many independent random variables with finite moments approaches a Gaussian random variable. Hence the coarse graining process causes the $f(\alpha)$ curves to look more and more Gaussian at scales larger than the pixel correlation lengths. Surprisingly, there is an optimum parameter value for generating the least random, most intermittent distribution. Based on the flatness $F$, the distributions for $\eta=0.02$ and $\eta=0.24$ are more nearly Gaussian than the relatively intermittent one for $\eta=0.2$, even though this does not have the strongest communication between cells. This appears to stem from competition between two effects. An intermittent distribution is characterized by enhanced strong-field tails. Communication among cells has two effects. First, the strong fields are spread out, which populates the distribution tails and increases intermittence. Second, however, the large field strengths are correspondingly reduced, which truncates the tails and reduces intermittence. The corresponding $f(\alpha)$ plots suggest that, in the context of the cellular dynamo model, the more intermittent the distribution the more it "looks" like a multifractal.

The optimum random cell dynamo image has a much larger cancellation exponent than solar images even though $\kappa$ is minimized in this case. In general the cancellation exponents seem to vary with randomness, and it is possible that essentially all of the small-scale cancellation in the solar images is associated with noise.

The simulated field from the ABC dynamo presents a singularity spectrum resembling that of the solar magnetic field. It is characterized by two parts: a multifractal that corresponds to large fields, and a cascade of extremely weak field peaks, apparently due to slight noise arising as an artifact of the analysis of the flow. The key characteristic of this ABC dynamo is its chaotic nature, including correlations on all length scales.

The general import of the above considerations seems to be that intermittence and chaos may underlie multifractal structure. When introducing communication among cells in the random cell dynamo, we found that for scales larger than the correlation length the coarse graining process drove the measure to a Gaussian via the Central Limit Theorem. Contrasting this result with that of the chaotic $A B C$ dynamo we conclude that a crucial ingredient is long range correlation. $\mathrm{Up}$ to this point we have been unsuccessful in introducing this mechanism into the "microscopic" dynamics of the cellular dynamo. It is possible that these long range effects can only be included via global mixing of the fields, such as in a "baker's transformation." The present work thus points the way for future investigations.

We want to thank D. Galloway for generously offering his data from the ABC dynamo simulation. We are also grateful to G. A. Chapman and S. R. Walton for acquiring and processing the San Fernando Observatory data, and to the anonymous referee for useful comments. One of the authors (ACC) wants to acknowledge the release time for this work provided by the Research and Grants Committee at California State University Northridge.

This work was supported in part by NSF grants ATM9309901, ATM-8817634, and ATM-9115111.

\section{REFERENCES}

Brandenburg, A., Procacia, I., Segel, D., \& Vincent, A. 1992, Phys. Rev. A, 46, 4819

Chapman, G. A., \& Walton, S. R. 1989, in High Spatial Resolution Solar Observations, ed. O. van der Luhe (Sunspot, NM: National Solar Obs.), 402 Chhabra, A. B., Meneveau, C., Jensen, R. V., \& Sreenivasan, K. R. 1989, Phys. Rev. A, 40, 5284

Du, Y., \& Ott, E. 1992, Univ. Maryland Preprint

Evertsz, C. J. G., \& Mandelbrot, B. B. 1992, in Chaos and Fractals: New Frontiers of Science, ed. H.-O. Peitgen, H. Jürgens, \& D. Saupe (Berlin: Springer), 921

Finn, J., \& Ott, E. 1988, Phys. Fluids, 31, 2992

Galloway, D. J., \& Frisch, U. 1986, Geophys. Astrophys. Fluid Dyn., 36, 53

Galloway, D. J., \& Proctor, M. R. E. 1992, Nature, 356, 691

Halmos, P. R. 1962, Measure Theory (New York: Van Nostrand)

Halsey, T. C., Jensen, M. H. Kadanoff, L. P., Procaccia, I., \& Schraiman, B. I. 1986, Phys. Rev. A, 33, 1141

Krause, F., \& Rädler, K. H. 1980, Mean-Field Magnetohydrodynamics and

Dynamo Theory (Berlin:Springer).

Lawrence, J. K., Chapman, G. A., \& Walton, S. R. 1991, ApJ, 375, 771

Lawrence, J. K., Ruzmaikin, A. A., \& Cadavid, A. C. 1993, ApJ, 417, 805

Martinez, V. J. 1991, in Applying Fractals in Astronomy, ed. A. Heck \& J. M. Perdang (Berlin: Springer), 135

Meakin, P. 1987, Phys. Rev. A, 36, 2833

Meneveau, C., \& Screenivasan, K. R. 1989, Phys. Lett. A, 137, 103

. 1991, J. Fluid Mech., 224, 429

Ott, E., Du, Y., Sreenivasan, K. R., Juneja, A., \& Suri, A. K. 1992, Phys. Rev. Lett., 69, 2654

Parker, E. N. 1979, Cosmic Magnetic Fields (Oxford: Oxford Univ. Press)

Poezd, A., Ruzmaikin, A. A., \& Sokoloff, D. D. 1992, Soviet Phys.—JETP, 101, 851

Ruzmaikin, A. A., Liewer, P., \& Feynman, J. 1994, Geophys. Astrophys. Fluid Dyn., in press

Ruzmaikin, A. A., Liewer, P., \& Feynman, J. 1994, Geophys. Astrophys. Fluid Dyn., in press

Ruzmaikin, A. A., Sokoloff, D. D., \& Tarbell, T. D. 1991, in The Sun and Cool Stars: Activity Magnetism, Dynamos, ed. I. Tuomien, D. Moss, \& G. Rüdiger (Berlin: Springer), 140

Title, A. M., Tarbell, T. D., Topka, K. P., Ferguson, S. H., Shine, R. A., \& the SOUP Team 1989, ApJ, 336, 475

Van de Water, W., \& Schram, P. 1988, Phys. Rev. A, 37, 3118

Zel'dovich, Ya. B., Ruzmaikin, A. A., \& Sokoloff, D. D. 1983, Magnetic Fields in Astrophysics (New York: Gordon and Breach) 